\title{
Direct measurement of the fine-structure interval in alkali atoms using diode lasers
}

\author{
Ayan Banerjee, Umakant D. Rapol, and Vasant Natarajan ${ }^{\mathrm{a})}$ \\ Department of Physics, Indian Institute of Science, Bangalore 560 012, India
}

\begin{abstract}
We demonstrate a technique for directly measuring the fine-structure interval in alkali atoms using two frequency-stabilized diode lasers. Each laser has a linewidth of order $1 \mathrm{MHz}$ and precise tunability: one laser is tuned to a hyperfine transition in the $D_{1}$ line, and the other laser to a hyperfine transition in the $D_{2}$ line. The outputs of the lasers are fed into a scanning Michelson interferometer that measures the ratio of their wavelengths accurately. To illustrate the technique, we measure the fine-structure interval in $\mathrm{Rb}$, and obtain a value of $237.6000(3)(5) \mathrm{cm}^{-1}$ for the hyperfine-free $5 P_{3 / 2}-5 P_{1 / 2}$ interval.
\end{abstract}

The advent of semiconductor diode lasers has revolutionized atomic physics and particularly the field of laser spectroscopy. ${ }^{1}$ Single-mode laser diodes with continuous wave output powers of few 10s of $\mathrm{mW}$ are now available at very low cost. By placing the diodes in an external cavity and using optical feedback from an angle-tuned grating, ${ }^{2}$ they can be made to operate at single frequency (single longitudinal mode) with linewidths of order $1 \mathrm{MHz}$ and tunability over several $\mathrm{nm}$. This has increased the access of researchers to atomic physics experiments which have earlier required expensive ring-cavity dye or Ti-sapphire lasers. For example, the field of laser cooling ${ }^{3}$ has blossomed in the past decade as several alkali atoms and alkalilike ions have cooling transitions in the infrared which are accessible with stabilized diode lasers. The main advantages of diode lasers are in terms of their tunability and narrow spectral width. In addition, techniques such as saturated-absorption spectroscopy using counter-propagating pump and probe beams to eliminate the first-order Doppler effect can help resolve narrow hyperfine transitions within a given atomic line. It is thus possible to get an absolute frequency calibration of the laser by locking to such a transition.

In this letter, we show that this well-established capability of diode lasers can be used in an important application: to make precise measurements of fine-structure intervals in alkali atoms. Knowledge of fine-structure intervals is important for several reasons, e.g., in the study of atomic collisions, astrophysical processes, and relativistic calculations of atomic energy levels. Alkali atoms are particularly important because of their widespread use in ultracold collision studies, photoassociation spectroscopy, atomic tests of parity violation, and more recently in Bose-Einstein condensation. The species we have studied is $\mathrm{Rb}$, but the technique is more general and should prove useful in other alkali atoms (such as $\mathrm{Li}, \mathrm{K}$, and $\mathrm{Cs}$ ), alkalilike ions, and indeed any system where the transitions are accessible with diode lasers. Our technique uses two diode lasers, one of which is tuned to the $D_{1}$ line and the other to the $D_{2}$ line. The output of the lasers is fed into a scanning Michelson interferometer to directly

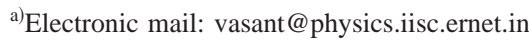

obtain their wavelength ratio. Using tabulated values ${ }^{4}$ for the wavelength of the $D_{2}$ line, we extract the $D_{1}-D_{2}$ splitting. The value we obtain for the $5 P$ state of $\mathrm{Rb}$ has a precision of 5 parts in $10^{8}$, and can be potentially improved by another order of magnitude.

The interferometer for the ratio measurement, shown schematically in Fig. 1, has been described extensively in a previous publication ${ }^{5}$ where we highlighted its use as a precision wavelength meter. For consistency with the terminology there, we will call the two lasers as "reference" and "unknown," respectively. The basic idea is to obtain the ratio of the two laser wavelengths using a scanning Michelson interferometer where both lasers traverse essentially the same path. As the interferometer is scanned, the interference pattern on the detectors goes alternately through bright and dark fringes and the ratio of the number of fringes counted is the ratio of the two wavelengths. The ratio obtained is a wavelength ratio in air, however, the wavelength ratio in vacuum (or equivalent frequency ratio) is easily calculated by making a small correction for the dispersion of air $^{6}$ between the reference wavelength and the unknown.

The fine-structure measurements are done with two diode laser systems. The first diode laser system (or reference laser) is built around a commercial single-mode diode (Mitsubishi ML60125R-01) with a nominal operating wavelength

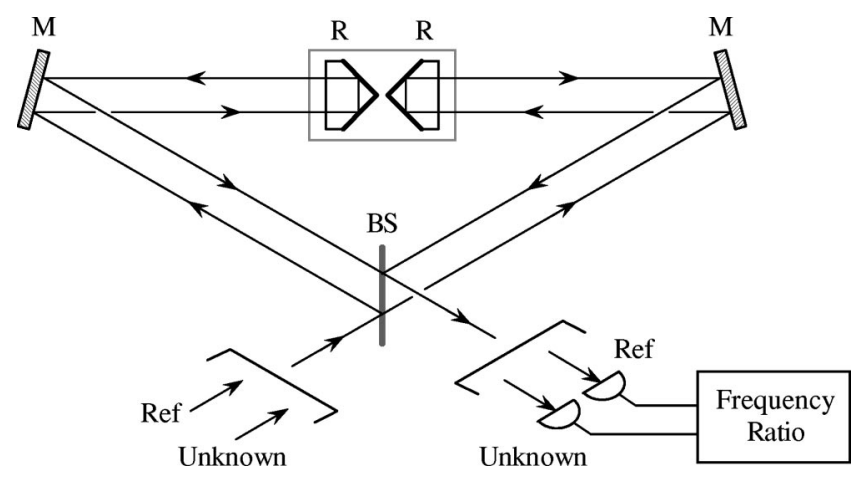

FIG. 1. Schematic of the scanning interferometer. The Michelson interferometer consists of a beamsplitter (BS), two end mirrors (M), and two retroreflectors $(\mathrm{R})$ mounted back to back on a movable cart. The ratio of the number of fringes counted in the two detectors for a given cart travel yields the ratio of the two wavelengths. 


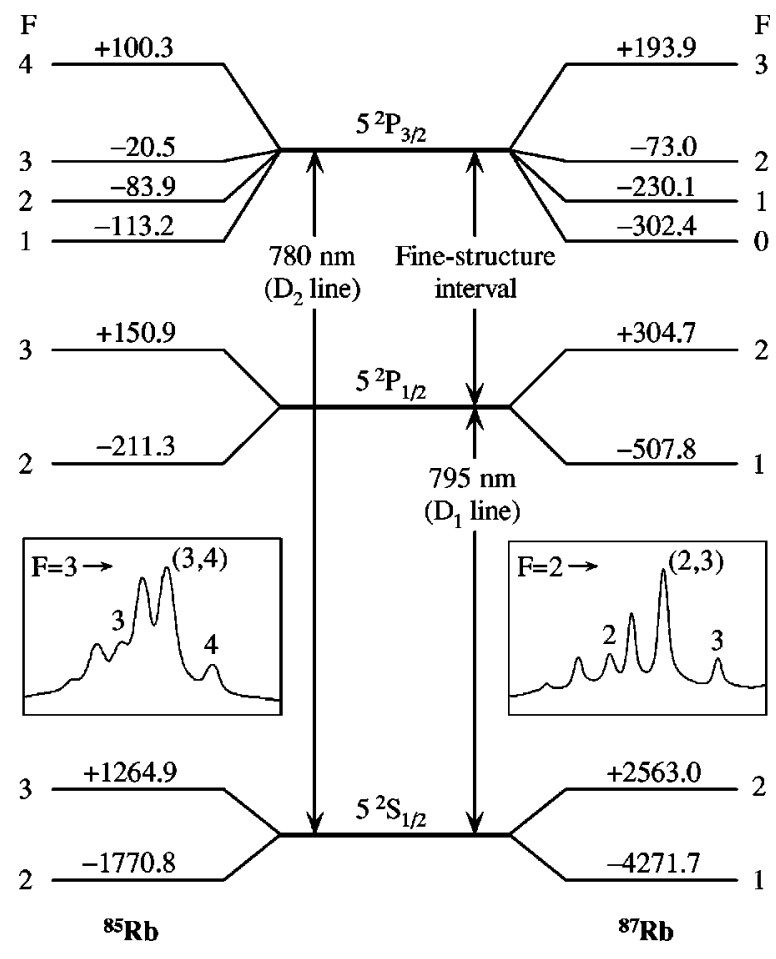

FIG. 2. Rb energy levels. Shown are the relevant energy levels of ${ }^{85} \mathrm{Rb}$ and ${ }^{87} \mathrm{Rb}$ in the ground $5 S$ state and first excited $5 P$ state. The various hyperfine levels are labeled with the value of the total angular momentum quantum number $F$, and the number on each level is the energy displacement (in $\mathrm{MHz}$ ) from the unperturbed state. The two insets show probe transmission as our diode laser is scanned across the $D_{2}$ line in a Doppler-free saturated absorption spectrometer. The inset on the left-hand side is for transitions starting from the $F=3$ state in ${ }^{85} \mathrm{Rb}$, and the one on the right-hand side is for transitions starting from the $F=2$ state in ${ }^{87} \mathrm{Rb}$.

of $785 \mathrm{~nm}$. The light is collimated using an aspheric lens to give an elliptic beam of $5.8 \mathrm{~mm} \times 1.8 \mathrm{~mm} 1 / e^{2}$ diameter. The laser is frequency stabilized in a standard external-cavity design (Littrow configuration) using optical feedback from a 1800 lines/mm diffraction grating mounted on a piezoelectric transducer. ${ }^{2}$ Using a combination of temperature and current control, the laser is tuned close to the $780 \mathrm{~nm} D_{2}$ line in atomic $\mathrm{Rb}\left(5 S_{1 / 2} \leftrightarrow 5 P_{3 / 2}\right.$ transition). A part of the output beam is tapped for Doppler-free saturated-absorption spectroscopy in a $\mathrm{Rb}$ vapor cell. The various hyperfine transitions (and crossover resonances) in the two common isotopes of $\mathrm{Rb},{ }^{85} \mathrm{Rb}$ and ${ }^{87} \mathrm{Rb}$, are clearly resolved, as shown in the insets of Fig. 2. The linewidth of the hyperfine peaks is $15-20 \mathrm{MHz}$; this is somewhat larger than the $6.1 \mathrm{MHz}$ natural linewidth and is primarily limited by power broadening due to the pump beam. ${ }^{7}$ The injection current into the laser diode is modulated slightly to obtain an error signal so that the laser can be locked to any of the peaks in the saturatedabsorption spectrum. The second diode laser system (unknown laser) is identical to the first one, except that the laser diode (SDL 5311-G1) has a nominal operating wavelength of $792 \mathrm{~nm}$. After stabilization, it is tuned to the $795 \mathrm{~nm} D_{1}$ line in atomic $\mathrm{Rb}\left(5 S_{1 / 2} \leftrightarrow 5 P_{1 / 2}\right.$ transition). The elliptic beams from the two lasers are fed into the Michelson interferometer. The large Rayleigh ranges $(\sim 34 \mathrm{~m}$ and $\sim 3 \mathrm{~m}$ in the two directions) ensure that the beams remain collimated over the length of the interferometer and diffraction effects are not significant.

For the fine-structure measurements, we lock the refer-
TABLE I. Listed are the measured wavelength ratios and $5 P$ fine-structure interval. The reference laser was locked to a transition of the $D_{2}$ line in ${ }^{85} \mathrm{Rb}$, corresponding to a frequency of $3.8422958 \times 10^{14} \mathrm{~Hz}$, while the second laser was tuned to various hyperfine transitions of the $D_{1}$ line in ${ }^{85} \mathrm{Rb}$ and ${ }^{87} \mathrm{Rb}$, as listed. The hyperfine-free interval in vacuum was extracted by first converting the wavelength ratio to a frequency ratio and then removing the hyperfine shifts shown in Fig. 2. The errors are statistical $1 \sigma$ deviations.

\begin{tabular}{lcc}
\hline \hline $\begin{array}{l}\text { Measured transition } \\
\left(D_{1} \text { line }\right)\end{array}$ & Wavelength ratio & $\begin{array}{c}5 P_{3 / 2}-5 P_{1 / 2} \\
\left(\mathrm{~cm}^{-1}\right)\end{array}$ \\
\hline${ }^{85} \mathrm{Rb}, F=2 \rightarrow F^{\prime}=3$ & $1.01888035(3)$ & $237.6001(4)$ \\
${ }^{85} \mathrm{Rb}, F=3 \rightarrow F^{\prime}=2$ & $1.01888954(4)$ & $237.6002(5)$ \\
${ }^{87} \mathrm{Rb}, F=1 \rightarrow F^{\prime}=2$ & $1.01887318(5)$ & $237.6002(6)$ \\
${ }^{87} \mathrm{Rb}, F=2 \rightarrow F^{\prime}=1$ & $1.01889379(3)$ & $237.5995(4)$ \\
\hline \hline
\end{tabular}

ence laser to the $5 S_{1 / 2}, F=3 \leftrightarrow 5 P_{3 / 2}, F^{\prime}=(3,4)$ crossover resonance in ${ }^{85} \mathrm{Rb}$, i.e., midway between the $F=3 \leftrightarrow F^{\prime}=3$ and the $F=3 \leftrightarrow F^{\prime}=4$ transitions. ${ }^{8}$ From the Rb energy-level tables ${ }^{4}$ and measured hyperfine shifts, ${ }^{9}$ this corresponds to a frequency of $3.8422958 \times 10^{14} \mathrm{~Hz}$. To get several independent measurements of the fine-structure splitting, we lock the second laser to different hyperfine transitions of the $D_{1}$ line in the two isotopes of $\mathrm{Rb}$. In each case, the wavelength ratio is measured about 100 times. The values are plotted as a histogram and a Gaussian fit to the histogram yields the mean ratio and the $1 \sigma$ (statistical) error in the mean. The mean wavelength ratios obtained from four independent measurements are listed in Table I. We extract the hyperfinefree fine-structure interval in vacuum by making two corrections to the measured ratio. First, we convert the wavelength ratio in air to a frequency ratio using the refractive index of air at the two wavelengths from Edlén's formula: ${ }^{6} n$ $=1.000275163$ at $780 \mathrm{~nm}\left(D_{2}\right)$ and $n=1.000275068$ at $795 \mathrm{~nm}\left(D_{1}\right)$. Then, we remove the hyperfine frequency shifts shown in Fig. 2, which are known to sub-MHz accuracy. ${ }^{9}$ The extracted values of the fine-structure interval are listed in Table I, from which we obtain an average value of:

\section{$237.6000(3)(5) \mathrm{cm}^{-1}$,}

with the quoted errors being statistical and systematic, respectively. Isotope shifts in the fine-structure interval are negligible at this level of precision.

The main source of statistical error is that the frequency counter counts zero crossings and does not count fractional fringes. The total number of fringes counted depends on the fringe rate (or cart speed) coupled with the $10 \mathrm{~s}$ integration time. Currently, our photodiode electronics limits the cart speed so that we can use only about $20 \mathrm{~cm}$ of cart travel per measurement. This results in a single-shot statistical error of about 5 parts in $10^{7}$ in each data set. ${ }^{10}$ The error of less than 5 parts in $10^{8}$ in Table I comes after averaging over $\sim 100$ individual measurements. Since the coherence length of the stabilized diode laser is about $50 \mathrm{~m}$, it should be possible to use at least $1 \mathrm{~m}$ of cart travel for each measurement with simple improvements in the counting electronics. This should help in reducing the statistical error in future to below $1 \mathrm{MHz}$.

There are several potential sources of systematic error, the main two being variation in the lock point of the lasers and nonparallelism of the two laser beams in the interferom- 
eter. We have checked for the first error by measuring the fine-structure interval with the unknown laser on different hyperfine transitions of the $D_{1}$ line. As seen from Table I, within the errors quoted, the different ratios yield consistent values for the hyperfine-free fine-structure interval. This implies that there is no significant variation in the lock point of the lasers. The second source of systematic error, namely that the two beams have a small angle between them, is more serious. Any misalignment would cause a systematic increase in the measured ratio given by $1 / \cos \theta$, where $\theta$ is the angle between the beams. We have tried to minimize this error in two ways. The first method is to use the unused output beam of the reference laser (the one on the same side of the beamsplitter as the input beam) as a tracer for aligning the unknown laser beam, and checking for parallelism over a distance of about $2 \mathrm{~m}^{11}$ The second method is to check for parallelism by looking for a minimum in the measured ratio as the angle of the unknown beam is varied. This works because the measured value is always larger than the correct value, whether $\theta$ is positive or negative, and becomes minimum when $\theta=0$.

To get an estimate of the systematic error using these methods of alignment, we have measured the frequency ratio of two identical stabilized diode lasers locked to different hyperfine transitions in the $D_{2}$ line of ${ }^{85} \mathrm{Rb},{ }^{12}$ as described in our earlier work. ${ }^{5}$ The frequency difference is known to be $2944 \mathrm{MHz}$, while the measurements yield a mean value of 2932(16) MHz. This shows that the systematic error is below $16 \mathrm{MHz}$ or about $0.0005 \mathrm{~cm}^{-1}$. In the future, this error can also be brought down to the sub-MHz level by carefully checking for parallelism over longer distances.

The value for the $5 P$ fine-structure interval we obtain can be compared to the value listed in the Rb energy-level tables published by the National Institute of Standards and Technology (NIST): ${ }^{4} 237.60 \mathrm{~cm}^{-1}$. Our result is consistent with this value but has significantly higher accuracy. The NIST value is obtained by fitting the energy levels to all available spectroscopic data. The accuracy of $0.01 \mathrm{~cm}^{-1}$ for this value is typical for most alkali atoms and our technique has the potential to improve this by two orders of magnitude. In general, fine-structure intervals are known with much less precision when compared to hyperfine shifts. This is because hyperfine shifts, which are of order $\mathrm{GHz}$, are more accessible to techniques such as microwave resonance or heterodyne measurements, ${ }^{13}$ while fine-structure splittings are of order $\mathrm{THz}$ and can not be measured very easily. It is in this range of frequency differences that our technique is uniquely suitable. Finally, we mention that the scanning interferometer can also be used as a precision wavelength meter, giving the unknown wavelength in terms of the reference. ${ }^{5}$ This could be important, for example, in laser cooling of ions where it is necessary to set the laser frequency with $\sim \mathrm{MHz}$ precision but not always possible to find transitions to which the cooling laser can be locked. Recently, the absolute frequency of a diode laser stabilized on the $\mathrm{Rb} D_{2}$ line has been measured with sub-MHz accuracy. ${ }^{13}$ Our wavelength meter can thus act as a secondary reference to improve the absolute precision of energy-level tables.

In conclusion, we have demonstrated a technique to directly measure the fine-structure interval in alkali atoms. Our method uses two stabilized diode lasers, one locked to the $D_{1}$ line and the other to the $D_{2}$ line, and a scanning Michelson interferometer to obtain their wavelength ratio. As an illustration of the power of this technique, we measure the interval in the $5 P$ state of $\mathrm{Rb}$ to be $237.6000(3)(5) \mathrm{cm}^{-1}$, or $7123069(9)(15) \mathrm{MHz}$. The method is easily extendable to other systems in which transitions are accessible with diode lasers. In addition, the interferometer with a diode laser locked to an atomic transition can be used as a precision wavelength meter for tunable lasers ${ }^{5}$ since the atomic transition acts as a convenient calibrated wavelength marker.

The authors are grateful to Dipankar Das and Stéphane Berciaud for help with the measurements. This work was supported by research grants from the Board of Research in Nuclear Sciences (DAE), and the Department of Science and Technology, Government of India.

${ }^{1}$ C. E. Wieman and L. Hollberg, Rev. Sci. Instrum. 62, 1 (1991).

${ }^{2}$ K. B. MacAdam, A. Steinbach, and C. Wieman, Am. J. Phys. 60, 1098 (1992); L. Ricci, M. Weidemüller,, T. Esslinger, A. Hemmerich, C. Zimmermann, V. Vuletic, W. König, and T. Hänsch, Opt. Commun. 117, 541 (1995).

3 For a review, see: S. Chu, Rev. Mod. Phys. 70, 685 (1998); C. N. CohenTannoudji, ibid. 70, 707 (1998); and W. D. Phillips, ibid. 70, 721 (1998).

${ }^{4}$ C. E. Moore, Natl. Stand. Ref. Data Ser. 35, 180 (1971).

${ }^{5}$ A. Banerjee, U. D. Rapol, A. Wasan, and V. Natarajan, Appl. Phys. Lett. 79, 2139 (2001).

${ }^{6}$ B. Edlén, Metrologia 2, 71 (1966).

${ }^{7}$ Collisional broadening at the gas pressure in the $\mathrm{Rb}$ vapor cell is estimated to be $\sim 10 \mathrm{kHz}$ and the linewidth of the laser has been independently measured to be $\sim 1 \mathrm{MHz}$.

${ }^{8}$ We lock to crossover resonances because they are more prominent than the hyperfine peaks.

${ }^{9}$ E. Arimondo, M. Inguscio, and P. Violino, Rev. Mod. Phys. 49, 31 (1977).

${ }^{10}$ The calculated error from missing one fringe over a distance of $20 \mathrm{~cm}$ is about 1 part in $10^{6}$ at $780 \mathrm{~nm}$. Our error is smaller because we are measuring a wavelength ratio close to 1 and chances are high that either both counters see a zero crossing or miss it.

${ }^{11}$ Ideally, the unknown beam should enter exactly where the reference beam exits so that both beams traverse exactly the same path, albeit in opposite directions. However, this causes one laser to feed into the other and destabilize it. Therefore, we align the beams with a small offset and check for parallelism over a finite distance.

${ }^{12}$ The reference laser was locked to the $F=3 \leftrightarrow F^{\prime}=(3,4)$ crossover resonance. The unknown laser was locked to the $F=2 \leftrightarrow F^{\prime}=(2,3)$ crossover resonance, whose frequency is $2944 \mathrm{MHz}$ higher (see Fig. 2).

${ }^{13}$ J. Ye, S. Swartz, P. Jungner, and J. L. Hall, Opt. Lett. 21, 1280 (1996). 